\title{
Ethical perspectives on the management of disorders of sex development in children
}

REVIEW

\author{
This article was published in the following Dove Press journal: \\ Medicolegal and Bioethics \\ 8 June 2015 \\ Number of times this article has been viewed
}

\author{
Breanna Lathrop ${ }^{1,2}$ \\ Teresa B Cheney ${ }^{3}$ \\ 'Good Samaritan Health Center, \\ Atlanta, GA, USA; ${ }^{2}$ South University, \\ Savannah, GA, USA; ${ }^{3}$ AppleCare \\ Immediate Care, LLC, Statesboro, \\ GA, USA
}

\begin{abstract}
The management of a child with disorders of sex development is complex from both a medical and sociocultural perspective. Decisions impacting the future sexual function and sex identity of the child are made on behalf of the child by their parents and health care providers. Such decisions are rarely straightforward and outcome data to guide practice are limited and conflicting. This manuscript explores the ethical issues present in prenatal diagnosis, sex assignment, medical management, and surgical intervention in the case of children with disorders of sex development. Suggestions for an ethics-based approach to management are offered including the use of multidisciplinary teams and guidelines, improved communication between providers and parents, the early and continued inclusion of mental health professionals, and the use of an independent review of treatment plans. The goal of an ethics-based management approach is the promotion of the autonomy and wellbeing of the affected child.
\end{abstract}

Keywords: intersex, disorders of sex development, ethics, decision-making

\section{Introduction}

The birth of a child initiates a transition period for the family in which others make critical decisions on behalf of the child. This experience becomes more challenging and stressful when unexpected medical decision-making is required. The case of intersex infants, children affected by disorders of sexual development, presents a unique, ethically charged decision-making dilemma. Parents, guardians, and the medical team supporting them must make decisions on behalf of the infant with the goal of promoting a well-adjusted adult who identifies and is happy with his or her assigned sex. ${ }^{1}$ The best management approach to accomplish this goal is both controversial and individual-specific. This manuscript provides an overview of the ethical challenges in the management of intersex conditions and suggestions for practice and research to promote the autonomy of the child into adulthood.

\section{Disorders of sex development}

Until the last decade, the term intersex was used to describe individuals born with ambiguous genitalia. The Intersex Society of North America defines intersex as conditions in which a person is born with sexual anatomy that is not congruent with typical definitions of female and male. ${ }^{2}$ In 2006, the Lawson Wilkins Pediatric Endocrine Society and European Society for Paediatric Endocrinology released a consensus report recommending the term disorders of sex development (DSD) be used to promote clinical clarification and patient respect. ${ }^{3}$ The consensus group thus defines DSD as
Correspondence: Breanna Lathrop 947 Custer Avenue SE, Atlanta, GA 303।6-3107, USA

Tel + I 6783622742

Email breanna.lathrop@gmail.com 
"congenital conditions in which development of chromosomal, gonadal or anatomical sex is atypical". ${ }^{3}$

DSD encompass a wide range of medical diagnoses which can be classified for improved clarification and management. Shomaker et al describe four types of DSD based on karyotyping and hormone sensitivity: chromosomal or gonadal differentiation disorders, hormone production disorders, abnormalities of internal ducts, and abnormalities in end organ response. ${ }^{4} \mathrm{~A}$ correct and timely diagnosis guides medical and surgical decision-making and identifies the need for immediate intervention such as in cases of adrenal crisis.

While the true incidence of DSD is unknown, approximately one in every 2,000 live births is affected. ${ }^{5,6}$ The dominant medical management approach to DSD has been surgical intervention with the intent of achieving normalization. This approach is based on the belief that intersex is pathological and devalues the experience of affected individuals. ${ }^{7}$ Activists have more recently objected against early genital surgery since there is evidence of lack of effectiveness of early surgery. ${ }^{8}$ Surgical outcome data are largely insufficient, ${ }^{3}$ and the evidence available is often conflicting. Given the complex and conflicting history of the management of DSD, this manuscript provides a review of published approaches and ethical considerations without presenting a specific guideline for management. The management of DSD must always be undertaken on a case-by-case basis with the goal of maximizing long-term physical and psychological outcomes for individuals with DSD.

\section{Decision-making perspectives}

The medical and surgical management of a child with DSD requires several major decisions to be made on behalf of the child. First, the child must receive a sex assignment. The process of sex assignment results in a decision to raise the child as male or female and is based on factors such as specific diagnoses, psychosocial factors, potential fertility, predicted sexual function, and minimizing physical risk. ${ }^{9}$ Next decisions are made regarding the management of the underlying condition which may or may not include surgical intervention. Understanding the perspectives of those involved in the decision-making process is paramount in creating strategies to promote an ethics-based decision-making process.

Parents have both an ethical responsibility and a right to promote and protect the interests of their child. ${ }^{10}$ As a result, the preferences of the parents in regard to sex assignment and medical/surgical management of their child are one of the most important factors in the decision-making process. Parents are most knowledgeable of the social and cultural environment in which the child will be raised and the ways in which these factors might influence sex assignment. However, the ability of parents to promote the wellbeing of their child may be inherently limited in situations of DSD. First, the complexity of DSD and the emotions attached to the diagnosis have the potential to limit the ethics-based decision-making process. Parents of infants with DSD have expressed difficulty conceptualizing the diagnosis of intersex, ${ }^{11}$ confusion due to poor communication with medical professions, ${ }^{12}$ and a high level of concern about the stigma attached to such a diagnosis. ${ }^{13}$ Anxiety, shock, and confusion may limit parents' abilities to obtain and correctly process the information they need to make fully informed decisions on behalf of their child. Ozbey and Etker describe parents as experiencing "gender panic" in an effort to nail down the sex of the child immediately, without full regard for the welfare of the child. ${ }^{14}$ Despite the efforts of a multidisciplinary team, some parents may not be able to process the abundance of information offered by the team, and therefore the parents may not be able to think beyond the immediate desire of surgical intervention. Sanders et al found the key motivating factor in parent behavior was the desire to protect their child from negative social or emotional outcomes. ${ }^{15}$ Parents felt that by making certain medical and surgical decisions they could protect their child. ${ }^{15}$ Early genital surgery was viewed as a means of protecting the child and providing clarity of the child's sex. ${ }^{11,12,15}$ Parents' emotional response may be mediated by cultural and religious factors as well. According to Rebelo et al, in some cultures such as the South African impoverished population, parents demand surgery at birth since the child would be treated socially and culturally as a freak. ${ }^{16}$ In their desire to establish the child's sex and protect their child from social stigma, parents risk prioritizing their immediate concerns over the long-term adult sexual function and potential fertility of the child. All management decisions made in early childhood by the parents have the potential to limit the child's autonomy to make their own management decisions in the future. Parents face the difficult task of balancing their need to protect their child emotionally and physically with the promotion of the child's future autonomy to make decisions about his/her sex and opportunities for sexual relationships.

In addition to their emotional response and desire to protect their child, parental decision-making may also be influenced by their personal desires for one sex over another. Such sex preference might be driven by personal preferences, family planning, or cultural influences. Warne and Raza reference the preference for males in many poor areas due to the difficulty of marrying an infertile daughter. ${ }^{17}$ 
These limitations highlight the need for a team-based approach to DSD decision-making and management. When the desires of the parents differ from the health care team, the role of the health care providers and a potential third party review becomes even more critical. The health care team must act as the child's advocate for the best outcome possible and may, in some cases, make the decision of sex assignment. An ideal outcome from this process would be future physical, functional, psychological, and surgical congruency for the child.

Health care professionals play a critical role in both educating parents and assisting in sex assignment decisionmaking while providing for the immediate medical needs of a DSD infant. Ozbey and Etker suggest that the diagnosis of DSD be made by a team consisting of at least a pediatric endocrinologist (as head of the team), geneticist, pediatric surgeon/urologist, and a pediatric psychologist. ${ }^{14}$ Shomaker et al describe the initial management of DSD as efficient information-gathering to aid in sex assignment, including immediate karyotyping and assessing for adrenal crisis. ${ }^{4}$ The first and foremost priority of the medical team is to promote the physical wellbeing of the infant and prevent complications such as adrenal crisis. When comparing stakeholders' concerns in the management of DSD, Kogan et al found that health care providers emphasized physical concerns to a higher degree when compared with advocates and parents. ${ }^{18}$ Parents and advocates expressed concerns related to sex and future body image. ${ }^{18} \mathrm{As}$ providers work with parents of DSD infants, they must be cognizant of this potential disconnect and assemble a multidisciplinary team able to address the emotional, social, and cultural concerns of these families.

Management of DSD is particularly challenging in that the affected child is unable to provide input at the time critical decisions are made. Sex assignment is a legal requirement and, in some cases, surgical decisions must be made early to prevent malignancy or severe urologic impairment. However, other surgical interventions have the potential to be delayed until the child is old enough to provide input. Advocacy organizations, most notably the Intersex Society of North America, advocate for sex assignment without surgery. ${ }^{8}$ They argue that parents' preferences often conflict with the best interest of the child. ${ }^{8}$ The preferences of children with DSD can only be assessed retroactively and literature assessing surgical outcomes and sex identity is limited and conflicting.

As evidenced above, the preferences and opinions of parents and health care providers are not always congruent and may not always be in the best interest of the child.
Early decisions made on behalf of the child may actually conflict with the child during the development of his or her sex and sexual identity. Given the complexity of DSD, Warne and Mann suggest the incorporation of an additional decision-making perspective. ${ }^{19}$ They suggest the inclusion of an independent body that reviews the case and treatment plan before any intervention to prevent bias. Such a review group may be particularly useful in situations where there is disagreement between health care team members or between providers and patients. However, careful evaluation and selection criteria must be developed to ensure the reviewers are both experts and unbiased in their decision-making. The ultimate goals of DSD management include a stable sex identity, optimal sexual function and body image, preservation of fertility, and minimizing intervention. ${ }^{20}$ The means by which to achieve these goals requires an intentional and ethicsbased evaluation and management approach. Considerations and strategies for such approaches will be discussed in the remainder of this manuscript.

\section{Prenatal management}

A child born with DSD represents an enormous challenge to parents and the health care team at the birth event. The ability to diagnose DSD prenatally, and ultimately provide necessary interventions during gestation, would be ideal for all concerned. Historically, ultrasound has been the technology of choice for the identification of sex in utero. However, children with DSD present complex challenges to the specification of sex identity, not only visually on ultrasound, but genetically and hormonally. Due to the complexity and wide differential of DSD, reliable prenatal diagnosis is limited, must be used with caution, and remains a challenge.

Colmant et al note that $3 \mathrm{D}$ ultrasound is valuable in the visual identification of ambiguous genitalia prenatally. ${ }^{21}$ Although safe, ultrasound has many limitations in regard to prenatal diagnoses of many forms of DSD, such as hypospadias. Colmant et al compared the sensitivity and specificity of ultrasound after 13 weeks gestation versus cellfree fetal DNA (cffDNA) extracted from the mother's blood in the first trimester to determine if fetal sex was correctly identified. ${ }^{21}$ Ultrasound was equal to cffDNA extraction in the ability to correctly diagnose fetal sex although ultrasound must wait until after the 13th week of gestation - the critical first trimester when the fetus is forming. Even genetic analysis has its limitations as some DSD cases were missed prenatally using cffDNA extraction. ${ }^{21}$

Emerging technological advances have allowed the identification of genetic mutations and the diagnosis of 
some forms of DSD during the prenatal course; however, the complexity of the human genome requires further exploration into the causes of DSD and potential medical interventions prenatally. ${ }^{22}$ The prenatal diagnosis and treatment of congenital adrenal hyperplasia, which causes genital ambiguity in females, is especially helpful through the use of genetic analysis. Congenital adrenal hyperplasia diagnosed at birth is considered an emergent condition, but early gestational molecular genetic analysis provides prenatal options to parents and to their female fetus, such as the use of dexamethasone administration during the course of the pregnancy, which prevents clitoral enlargement and labial fusion. ${ }^{23}$

Prenatal diagnosis of DSD is in its infancy, and more evidence-based studies are needed to diagnose DSD during early fetal development as well as research into medical therapies for treatment during gestation, and comparisons of postnatal and long-term outcomes of the child. To date, prenatal diagnosis of DSD "should focus on excluding conditions that lead to multiple anomalies and/or cognitive disabilities". ${ }^{24}$

\section{Postnatal management Sex assignment}

The care of an infant affected by DSD begins with sex assignment. While many aspects of medical management are controversial, there is general agreement that all DSD infants should be assigned a sex as soon as clinical data are available. ${ }^{14}$ However, sex assignment is not clear cut in the majority of cases. ${ }^{9}$ Factors influencing sex assignment include diagnosis, potential for fertility, appearance of genitalia, adult sexual function, surgical options, hormone replacement options, family preferences, and cultural factors. ${ }^{4,8,9}$ A multidisciplinary team is needed to gather information and present results and options to the family.

\section{Surgical intervention}

Surgical intervention may or may not be included in the management of DSD and is one of the most controversial aspects of management. Surgical intervention during infancy has been included in standard DSD management protocols ${ }^{25-27}$ and only more recently questioned. Surgical intervention was initially thought to be essential to the successful development of sex identity. ${ }^{7}$ This belief was possibly based on the hypothesis that sex identity is programmed during the first few years of life through social conditioning. ${ }^{20}$ Physiologic considerations which may support early surgery include satisfactory cosmetic results, reduction of urinary tract infections, and the maternal estrogenic effect on infant tissue. ${ }^{28}$
With advancements in diagnosis and treatment options as well as new understandings of sex identity, there has been a move away from early surgical intervention. In their survey of 60 DSD centers in 23 European nations, Pasterski et al found $52 \%$ reporting a decrease in the number of clitoroplasties performed. $^{29}$

Long-term outcome data on infants who have undergone surgical intervention are extremely limited..$^{5}$ The majority of studies address surgical outcomes for feminizing genitoplasty on women with congenital adrenal hyperplasia (CAH). Studies highlight both physiological and psychological outcomes. One UK study of DSD females found that women who had undergone early surgical intervention reported higher rates of non-sensuality and inability to orgasm than those who had not undergone surgery. ${ }^{6}$ This finding was consistent with a small study of women with CAH post-surgery. ${ }^{26}$ Of the six patients assessed, all had abnormal sensation to the clitoris. ${ }^{26}$ Krege et al examined 27 patients with CAH who underwent genitoplasty surgery from 1972 to 1978 and found $36 \%$ to have vaginal stenosis and subsequent anxiety about sexual relationships. ${ }^{30}$ Interestingly, the majority of women without vaginal stenosis also reported anxiety about sexual intimacy. ${ }^{30}$ In their assessment of 60 Middle Eastern women with $\mathrm{CAH}$, Seyam et al report that while cosmetic appearance of the genitalia was good to excellent in all patients, most were not married and did not report sexual relationships. ${ }^{31}$ Creighton et al assessed 44 adolescents with $\mathrm{CAH}$ who had undergone previous feminizing surgery. ${ }^{32}$ The cosmetic result was judged as poor in $41 \%$ and $98 \%$ would need additional surgery for improved cosmetic appearance, tampon use, or sexual intercourse. ${ }^{32}$ Davies et al report high complication rates following vaginal construction surgery including vaginal stenosis and fistula formation. ${ }^{33}$

There are several important caveats to the assessment of long-term outcomes. First, there is a distinct lack of studies comparing the outcomes between individuals of the same underlying DSD condition who did and did not undergo surgery. While complications and unsatisfactory results are reported following genitoplasty, there is also a lack of evidence on the outcomes associated with delaying or avoiding surgery. As Rangecroft explained, there is no evidence to demonstrate the long-term outcomes of rearing a severely virilized female without surgical intervention. ${ }^{25}$ Without comparison studies, attribution of negative outcomes to surgical procedures is difficult. For example, in their study of 91 women with DSD, Callens et al found that $40 \%$ reported sexuality-related distress. ${ }^{27}$ Sixty-four percent of women in the study had undergone feminizing surgery while the rest had not. ${ }^{27}$ Callens et al found 
no major differences in cosmetic or psychosexual outcomes between the groups of women. ${ }^{27}$ Without evidence for improved long-term outcomes without surgery, there is little evidence on which parents, health care providers, or other consulted parties can base surgical decisions.

A second limitation of the literature on surgical outcomes is the lack of standardization in the measurement of cosmetic and functional outcomes. Crouch and Creighton point out that most studies of feminizing surgery outcomes focus on appearance and the outcomes are often rated by individuals involved in the original procedure. ${ }^{34}$ Women's perspectives of functionality and appearance may also differ from those of the examiners. A vagina with the capacity for penile intercourse might be rated as a good outcome but if the woman experiences pain and lack of sensation during intercourse, she may not perceive this as a satisfactory outcome.

Given the limitations of current data on long-term surgical outcomes, especially in comparison to delayed surgery, it is not surprising that international guidelines and position statements differ in their handling of surgical intervention. The Intersex Society of North America argues that surgery reinforces stigmas against sexual differences, can damage sexual sensation, and may create a sex assignment not concurrent with future sex identity. ${ }^{8}$ Delay of surgery promotes the autonomy of the child's ability to make future decisions regarding sex identity and genital appearance. Warne and Mann question the capability of parents to consent to surgery when its effects on identity, fertility, and sexual function cannot be known until adulthood. ${ }^{19}$ The shift toward delaying or avoiding surgery as a means of promoting the autonomy and wellbeing of the child has gained international momentum. However, most published guidelines and position statements leave room to pursue surgery in infancy and early childhood. The CARES Foundation for CAH does not endorse any specific action regarding surgical intervention but recommends surgery be avoided until the child is medically stable, the parents are fully informed of the risks and benefits, and an expert surgeon is found. ${ }^{35}$ The Association of Paediatric Surgeons Working Party on the Surgical Management of Children Born with Ambiguous Genitalia states that infants with CAH should "not be denied what is currently standard treatment- that is, early feminizing surgery". ${ }^{25}$ The Halifax Resolution, adopted by the Fifth World Congress on Family Law and Children's Rights in 2009, provides principles to guide surgical decisions among which include leaving options open for the future and respecting the desires of parents. ${ }^{10}$ These principles may conflict and the resolution offers little guidance on how to prioritize principles.

\section{Mental health considerations}

The Intersex Society of North America advocates for mental health care as a critical component of DSD management. ${ }^{8}$ Counseling should begin immediately upon birth, and prenatally if possible, for the parents of infants with DSD and persist as an ongoing essential component of the child's care as appropriate. Data regarding mental health indicators among individuals with DSD, such as stress, sex identity, and relationships, present mixed findings. One study of 66 adolescents and 110 adults found that sex dysphoria is uncommon among individuals with DSD. ${ }^{36}$ However the same study found that only $25 \%$ of adults reported ever having a love relationship. Another study of children with DSD between the ages of 5 and 10 described children's selfreported physical quality of life to be close to that of unaffected children while their psychological quality of life was lower than unaffected children. ${ }^{28}$ Schutzmann et al compared women with DSD against women with and without a history of sexual and physical abuse. ${ }^{37}$ They found that women with DSD indicated higher psychological distress than the norm population and reported prevalence rates of self-harming behaviors and suicidal tendencies comparable to abused women. ${ }^{37}$ These studies point to the need for counseling and mental health care to be an integral part of DSD management throughout the life of an affected individual. Sex identity and satisfaction with sexual functioning is a core component of the human experience. Therefore, the social and psychological impact of DSD on the child is a critical part of the core of DSD management.

\section{Ethical principles and guidelines}

International efforts have been made to provide ethical principles and guidelines to improve the care of individuals with DSD. ${ }^{3,10}$ Given the complexity of the diagnosis, there is not a single sex assignment or treatment plan that best promotes the interests of the child. Rather, each DSD case must be considered on an individual basis ${ }^{14}$ within the context of the diagnosis and the family and cultural setting.

Several authors have offered principles to guide the management of DSD. Some principles focus on outcomes. For example, Houk and Lee recommend that the basic principles to guide treatment in all DSD cases are to "attempt to provide for fertility and sexual relations while minimizing health and psychosocial risks". ${ }^{9}$ While helpful, parents and health care providers may be unsure of and/or disagree about which actions promote these particular outcomes. Other authors describe an ethics-based approach to decision-making in DSD cases. The Halifax Resolution, adopted by the Fifth 
World Congress on Family Law and Children's Rights in 2009, provides six principles to guide decisions regarding the type and timing of surgery for children with DSD.

These six principles are: minimizing physical risk, minimizing psychological risk, preserving or promoting the capacity for satisfying sexual relationships, leaving options open for the future, and respecting parents' wishes and beliefs. ${ }^{10}$ Those involved in the decision-making process should consider each principle in order to thoroughly evaluate surgical options, including the delay or avoidance of surgery. The 2006 consensus statement on management of intersex disorders provides a long-term management strategy. ${ }^{3}$ Sponsored by the Lawson Wilkins Pediatric Endocrine Society and the European Society for Paediatric Endocrinology, the statement is drawn from the input of 50 international experts in DSD management. All management plans should include: expert evaluation of newborns before sex assignment, total evaluation and management performed by an experienced multidisciplinary team, sex assignment for all individuals, open communication with patients and families, and respect and confidentiality for patient and family concerns. ${ }^{3}$ The German Network DSD/ intersex offers three guiding ethical principles which include: fostering the wellbeing of the child and future adult, upholding the rights of children to participate in decisions, and respecting the parent-child relationship. ${ }^{38}$ The primary limitation of principle-based approaches is the difficulty in managing conflicting principles and decisions regarding how to value and balance each. While a clear hierarchy of principles would seem helpful, Johnston cautions against relying on a single ethical principle in decisions regarding the surgical management of atypical genitalia. ${ }^{39}$ She advises that decision-makers should use multiple principles within their social context to arrive at decisions.

While full implementation of these guidelines and principles should be the case for all DSD cases, their implementation is largely limited to the economically developed countries. Worldwide, poverty is the number one determinant of care received by individuals with DSD. ${ }^{17}$ Rebelo et al highlight the lack of follow-up care, non-existence of support groups, and parental requests for quick sex assignment to reduce stigma as challenges to DSD management in impoverished areas. ${ }^{16}$ With a lack of diagnostic tools and specialists, the final diagnosis is often guess work. ${ }^{17}$ Social stigma and cultural sex roles also influence sex assignment. In order for ethics-based DSD management approaches to be implemented in resource-poor areas, diagnostic tools, essential medicines, and specialists must be available.

\section{Toward an ethical approach}

Medical and surgical management for DSD is complex both physically and socially. The continued efforts of the medical community to provide care which is based on medical evidence and ethical principles is essential in promoting positive outcomes for individuals with DSD. To date, literature suggests several key strategies in promoting ethical care.

First, DSD management should be conducted by a multidisciplinary team under the direction of ethics-based guidelines. The importance of the multidisciplinary team is highlighted throughout the DSD literature. ${ }^{3,5,14}$ This team should include medical experts as well as social workers and mental health professionals. Much improvement has already been made in this area. In a survey of 60 DSD centers, $57.6 \%$ provided almost all of the subspecialties recommended in the 2006 Consensus Statement. ${ }^{29}$ To maximize the effectiveness of these teams, medical institutions can develop guidelines based on best medical practices as well as published principles such as the consensus guidelines ${ }^{3}$ and the Halifax Resolution. ${ }^{10}$ The authors of this paper have previously published a tool for guiding surgical decisions and applied the tool to the case of infants with DSD. ${ }^{40}$ The purpose of developing tools, guidelines, and/or standards of care is not to arrive at group policies, but rather to encourage and assist medical teams in the consideration of all aspects in the decision-making process and to avert biases.

Attention to the principle interests of children with DSD may also be promoted through improved communication between health care providers and parents. As highlighted in the work of Sanders et al, parents' decisions were primarily driven by their desire to protect their child. ${ }^{12}$ In their efforts to provide for the immediate wellbeing of their infant, they may neglect to consider critical outcomes such as future fertility and sexual functioning. Medical advancements allow providers the increased ability to advise parents about sex assignment based on adult outcomes rather than the appearance of genitalia at birth. ${ }^{4}$ Medical professionals face the difficult task of helping parents understand the lasting impact of early decisions and assisting parents in understanding their ability to assign sex with or without surgical intervention. Sanders et al found that parents wanted clear and complete information and did not want other health care professionals informed without their explicit permission. ${ }^{12}$ In their interviews with parents of children with DSD, Duguid et al found that parents wanted a steady, gradual stream of honest information. ${ }^{13}$ As addressed earlier in the manuscript, shock, confusion, and lack of experience limit parents' ability to receive information even when the 
health care team is making substantial efforts to engage in clear communication. Additional research should focus on communication aids and strategies to promote parental comprehension and understanding in order to better equip health care providers with this challenging task. While health care providers are first and foremost responsible for the health and wellbeing of the child, promotion of education and the protection of confidentiality can improve the parents' trust and their ability to make informed and ethical decisions.

A review of the literature highlights the need for early and continued inclusion of mental health professionals in all DSD cases. The Intersex Society of North America describes the licensed mental health professional as a central member of the multidisciplinary team. ${ }^{8}$ Parents need immediate counseling with continued family counseling, and the child should be provided with mental health services as individually appropriate. Mental health professionals can aid in assessing parents' response to information received and readiness for decision-making.

Management plans for DSD are particularly challenging in that decisions are made without complete knowledge of future outcomes such as fertility, sexual functioning, and sex identity. Social, cultural, and religious biases can cloud the judgment of parents and health care professionals even when multidisciplinary teams and ethical guidelines are in place. Warne and Mann argue that providers and parents are too intimately involved to make decisions which are in the best interest of the child. ${ }^{19}$ However, their intimate involvement also provides critical insight into the success of any management plan. Warne and Mann recommend that in addition to the preferences of families and providers, an "independent impartial assessment" be conducted for each case. ${ }^{19}$ An independent assessment offers protection against bias in cases in which parental preferences do not align with the best interests of the child. This type of assessment might be carried out by a medical ethicist, a government review board, or a medical team with expertise in the area but not directly involved with the case at hand. The feedback of such a review might also help providers and parents identify considerations they mistakenly overlooked in their decision-making process. However, the effectiveness of an independent review is limited by the absence of evidence regarding long-term surgical outcomes. Additional research is needed comparing physical and psychological outcomes with and without early surgical intervention. Such information is critical to the ability of all decision-makers to make decisions in the best interest of the child.
Finally, care for individuals with DSD must continue to evolve to allow for more successful maintenance of the autonomy of the individual. As described in the Halifax Resolution, the principle of keeping options open for the future recognizes that early decisions made on behalf of the child are not certain and promotes the right of the patient to participate in decision making. ${ }^{10}$ The German Network DSD/intersex further advocates that children themselves be involved in the decision-making process. ${ }^{38}$ Individuals with DSD should have the autonomy to make decisions about their body including their sex, relationships, sexuality, and physical appearance. Increased research on the lived experiences of individuals with DSD and long-term outcomes, both physical and emotional, may also increase the insight of parents and providers regarding the needs and desires of individuals with DSD.

Ethically challenging decisions are commonplace in health care, but few situations equal the management of infants with DSD in terms of complexity, stigma, and significance for the child's future. Advancements in diagnostic technology and medical care offer the potential improvement of DSD management but cannot substitute for ethical decision-making. Multidisciplinary teams using ethics-based guidelines, improved communication with and education of parents, the early and continued inclusion of mental health professionals, the use of an independent review of treatment plans, and an overarching priority of promoting the autonomy of the affected individual hold the potential for improving the wellbeing of individuals with DSD over their lifetime.

\section{Disclosure}

The authors have no conflicts of interest to disclose.

\section{References}

1. Gollu G, Yildiz RV, Bingol-Kologlu M, et al. Ambiguous genitalia: An overview of 17 years' experience. J Pediatr Surg. 2007;42(5): 840-844.

2. Isna.org [homepage on the Internet]. What is intersex. Intersex Society of North America; 1993-2008. Available from: http://www.isna.org/faq/ what_is_intersex. Accessed December 1, 2014.

3. Hughes IA, Houk C, Ahmed SF, Lee PA; Lawson Wilkins Pediatric Endocrine Society/European Society for Paediatric Endocrinology Consensus Group. Consensus statement on management of intersex disorders. J Pediatr Urol. 2006;2(3):148-162.

4. Shomaker K, Bradford K, Key-Solle M. The infant with ambiguous genitalia the pediatrician's role. Contemp Pediatr. 2009;26(4):40-56.

5. Maharaj NR, Dhai A, Wiersma R, Moodley J. Intersex conditions in children and adolescents: surgical, ethical, and legal considerations. J Pediatr Adolesc Gynecol. 2005;18(6):399-402.

6. Minto CL, Liao LM, Woodhouse CR, Ransley PG, Creighton SM. The effect of clitoral surgery on sexual outcome in individuals who have intersex conditions with ambiguous genitalia: a cross-sectional study. Lancet. 2003;361(9365):1252-1257. 
7. Preves SE. Sexing the intersexed: an analysis of sociocultural responses to intersexualtiy. Signs. 2002;27:523-556.

8. Chase C. What is the agenda of the intersex patient advocacy movement? Endocrinologist. 2003;13(3):240-242.

9. Houk CP, Lee PA. Approach to assigning gender in 46, XX congenital adrenal hyperplasia with male external genitalia: replacing dogmatism with pragmatism. J Clin Endocrinol Metab. 2010;95(10):4501-4508.

10. Gillam LH, Hewitt JK, Warne GL. Ethical principles for the management of infants with disorders of sex development. Horm Res Paediatr. 2010;74(6):412-418.

11. Gough B, Weyman N, Alderson J, Butler G, Stoner M. 'They did not have a word': the parental quest to locate a 'true sex' for their intersex children. Psychol Health. 2008;23(4):493-507.

12. Sanders C, Carter B, Goodacre L. Searching for harmony: parents' narratives about their child's genital ambiguity and reconstructive genital surgeries in childhood. J Adv Nurs. 2011;67(10):2220-2230.

13. Duguid A, Morrison S, Robertson A, et al. The psychological impact of genital anomalies on the parents of affected children. Acta Paediatr. 2007;96(3):348-352.

14. Ozbey H, Etker S. Disorders of sexual development in a cultural context. Arab J Urol. 2013;11(1):33-39.

15. Sanders C, Carter B, Goodacre L. Parents need to protect: Influences, risks and tensions for parents of prepubertal children born with ambiguous genitalia. J Clin Nurs. 2012;21(21-22):3315-3323.

16. Rebelo E, Szabo CP, Pitcher G. Gender assignment surgery on children with disorders of sex development: a case report and discussion from South Africa. J Child Health Care. 2008;12(1):49-59.

17. Warne GL, Raza J. Disorders of sex development (DSDs), their presentation and management in different cultures. Rev Endocr Metab Disord. 2008;9(3):227-236.

18. Kogan BA, Gardner M, Alpern AN, et al. Challenges of disorders of sex development: diverse perceptions across stakeholders. Horm Res Paediatr. 2012;78(1):40-46.

19. Warne GL, Mann A. Ethical and legal aspects of management for disorders of sex development. J Paediatr Child Health. 2011;47(9): 661-663.

20. Ahmed SF, Morrison S, Hughes IA. Intersex and gender assignment; the third way? Arch Dis Child. 2004;89(9):867-850.

21. Colmant C, Morin-Surroca M, Fuchs F, Fernandez H, Senat MV. Non-invasive prenatal testing for fetal sex determination: is ultrasound still relevant? Eur J Obstet Gynecol Reprod Biol. 2013;171(2): 197-204.

22. Arboleda VA, Sandberg DE, Vilain E. DSDs: genetics, underlying pathologies and psychosexual differentiation. Nat Rev Endocrinol. 2014;10(10):603-615.

23. Nimkam S, New MI. Prenatal diagnosis and treatment of congenital adrenal hyperplasia. Horm Res. 2007;67(2):53-60.

24. Adam MP, Fechner PY, Ramsdell LA, et al. Ambiguous genitalia: what prenatal genetic testing is practical? Am J Med Genet A. 2012;158A(6): 1337-1343

25. Rangecroft L; British Association of Paediatric Surgeons Working Party on the Surgical Management of Children Born With Ambiguous Genitalia. Surgical management of ambiguous genitalia. Arch Dis Child. 2003;88(9):799-801.

Medicolegal and Bioethics

\section{Publish your work in this journal}

Medicolegal and Bioethics is an international, peer-reviewed, open access journal exploring the application of law to medical and drug research and practice and the related ethical and moral considerations. The journal is characterized by the rapid reporting of reviews, case reports, guidelines and consensus statements, original research
26. Crouch NS, Minto CL, Laio LM, Woodhouse CR, Creighton SM. Genital sensation after feminizing genitoplasty for congenital adrenal hyperplasia: a pilot study. BJU Int. 2004;93(1):135-138.

27. Callens N, van der Zwan YG, Drop SL, et al. Do surgical interventions influence psychosexual and cosmetic outcomes in women with disorders of sex development? ISRN Endocrinol. 2012;2012:276742.

28. Crawford LJ, Warne G, Grover S, Southwell BR, Huston JM. Results from a pediatric surgical Centre justify early intervention in disorders of sex development. J Pediatr Surg. 2009;44(2):413-416.

29. Pasterski V, Prentice P, Hughes IA. Consequences of the Chicago consensus on disorders of sex development (DSD): current practices in Europe. Arch Dis Child. 2010;95(8):618-623.

30. Krege S, Walz KH, Hauffa BP, Körner I, Rübben H. Long-term follow-up of female patients with congenital adrenal hyperplasia from 21-hydroxylase deficiency, with special emphasis on the results of vaginoplasty. BJU Int. 2000;86(3):258-259.

31. Seyam RM, Bissada NK, Abdul-Aaly M, Sakati NA, Al Taweel W, Alkhudair WK. Long-term outcome of genital reconstruction of Middle Eastern women with congenital adrenal hyperplasia. Urol Ann. 2013;5(4):277-282.

32. Creighton SM, Minto CL, Steele SJ. Objective cosmetic and anatomical outcomes at adolescence of feminizing surgery for ambiguous genitalia done in childhood. Lancet. 2001;14:358(9276):124-125.

33. Davies MC, Creighton SM, Woodhouse CR. The pitfalls of vaginal construction. BJU Int. 2005;95(9):1293-1298.

34. Crouch NS, Creighton SM. Long-term functional outcomes of female genital reconstruction in childhood. BJU Int. 2007;100(2):403-407.

35. CARES Foundation [homepage on the Internet]. Moody M, Leight K. Ember L, Rink RC. Surgical Considerations for Girls with Classical $C A H$. CARES Foundation. Available from: http://www.caresfoundation. org/dosing/surgery/. Accessed March 26, 2015.

36. Jurgensen M, Kleinemeier E, Lux A, et al. Psychosexual development in adolescents and adults with disorders of sex development-results from the German Clinical Evaluation Study. J Sex Med. 2014;10(11): 2703-2714.

37. Schutzmann K, Brinkmann L, Schacht M, Richter-Appelt H. Psychological distress, self-harming behavior, and suicidal tendencies in adults with disorders of sex development. Arch Sex Behav. 2009;38(1): 16-33.

38. Wiesemann C, Ude-Koeller S, Sinnecker GH, Thyen U. Ethical principles and recommendations for the medical management of differences of sex development (DSD)/intersex in children and adolescents. Eur J Pediatr. 2010;169(6):671-679.

39. Johnston J. Normalizing atypical genitalia: how a heated debate went astray. Hastings Cent Rep. 2012;42(6):32-44.

40. Lathrop BL, Cheney TB, Hayman AB. Ethical decision-making in the dilemma of the intersex infant. Issues Compr Pediatr Nurs. 2014;37(1): 25-38.

\section{Dovepress}

and surveys. The manuscript management system is completely online and includes a very quick and fair peer-review system. Visit http://www.dovepress.com/testimonials.php to read real quotes from published authors. 